\title{
Sensitivity and Specificity of the Functional Hallux Limitus Test to Predict Foot Function
}

\author{
Craig Payne, DipPod, MPH ${ }^{\star}$ \\ Vivienne Chuter, BPod(Hons)† \\ Kathryn Miller, BPodł
}

Functional hallux limitus is an underrecognized entity that generally does not produce symptoms but can result in a variety of compensatory mechanisms that can produce symptoms. Clinically, hallux limitus can be determined by assessing the range of motion available at the first metatarsophalangeal joint while the first ray is prevented from plantarflexing. The aim of this study was to determine the sensitivity and specificity of this clinical test to predict abnormal excessive midtarsal joint function during gait. A total of 86 feet were examined for functional hallux limitus and abnormal pronation of the midtarsal joint during late midstance. The test had a sensitivity of 0.72 and a specificity of 0.66 , suggesting that clinicians should consider functional hallux limitus when there is late midstance pronation of the midtarsal joint during gait. (J Am Podiatr Med Assoc 92(5): 269-271, 2002)

Motion at the first metatarsophalangeal joint in the sagittal plane is essential for normal function. Structural hallux limitus is considered present when there is less than the assumed $65^{\circ}$ of dorsiflexion at the first metatarsophalangeal joint. Functional hallux limitus was described by Dananberg ${ }^{1}$ as the functional inability of the proximal phalanx of the hallux to extend on the first metatarsal head. Full range of motion is available at the joint during the nonweightbearing examination, and the inability of the hallux to dorsiflex is considered to occur only during gait. This may be only a momentary restriction and may be invisible to the naked eye. This momentary blockade to sagittal plane motion at the first metatarsophalangeal joint occurs late in the stance phase, as

*Lecturer, Department of Podiatry, School of Human Biosciences, La Trobe University, Bundoora, Victoria 3083, Australia.

$\dagger$ Postgraduate student, Department of Exercise and Sports Science, University of Sydney, Sydney, New South Wales, Australia.

$¥$ Research Assistant, Department of Podiatry, School of Human Biosciences, La Trobe University, Bundoora, Victoria, Australia. heel lift is being initiated by the pull of the advancing body when motion is needed at this joint. If the first metatarsophalangeal joint is unable to provide an adequate range of dorsiflexion, even if it is only a temporary block, the motion that is needed can theoretically occur by a variety of compensatory mechanisms, such as early heel-off or excessive pronation at the midtarsal joint. ${ }^{2-6}$ These compensatory mechanisms can result in a variety of pathologic symptoms. ${ }^{6,7}$

Clinically, the determination of functional hallux limitus is difficult, as there are generally no symptoms at the joint. It is necessary to observe the compensatory phenomena that are assumed to occur with functional hallux limitus. These can include lateral deviation of the center of pressure (determined by the in-shoe plantar pressure measurement), increased pronation at the midtarsal and subtalar joints (to use the dorsiflexion component at these joints), an abductory twist (so that the body can move weight around the blocked joint), early heel-off, and lack of full extension of the knee during propulsion. ${ }^{1,4}$ Dananberg $^{6}$ described a clinical test in which the range of motion of the first metatarsophalangeal joint 
is determined while the first metatarsal head is prevented from plantarflexing. If no range of motion is available, functional hallux limitus is considered likely.

No information is available on the prevalence of the assumed compensatory mechanisms for functional hallux limitus when it is considered present. The aim of this study was to determine the sensitivity and specificity of the nonweightbearing clinical test for functional hallux limitus to predict abnormal pronation at the midtarsal joint.

\section{Methods}

Subjects were recruited from the undergraduate student population at La Trobe University. Inclusion criteria were the absence of any significant orthopedic or structural deformities of the foot and lower limb and the absence of any foot pain.

Two clinicians examined each subject by using the functional hallux limitus test as described by Dananberg $^{6}$ (Fig. 1). In this nonweightbearing test, the examiner holds the foot of the subject in the subtalar joint neutral position. One hand is used to hold the first ray in a dorsiflexed position by loading the first metatarsal head. The other hand is used to dorsiflex the proximal phalanx of the hallux. The test result for functional hallux limitus was considered positive if there was immediate plantarflexion of the first metatarsal and negative if there was a delay in plantarflexion or a range of motion of the proximal phalanx on the first metatarsal before plantarflexion of the first ray occurred.

Two different clinicians then performed a visual gait analysis by observing the subject from the lateral

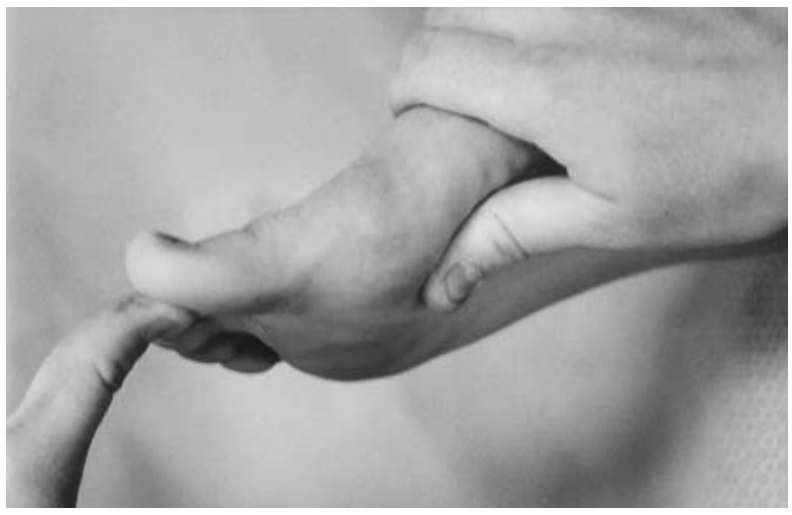

Figure 1. In the functional hallux limitus test, the examiner uses one hand to hold the first ray in a dorsiflexed position by loading the first metatarsal head and the other hand to dorsiflex the proximal phalanx of the hallux. view to determine abnormal midtarsal joint function (Fig. 2). A pen was used to mark the navicular tuberosity to aid visualization. Abnormal midtarsal joint function was considered to be present if the navicular was observed to move in a plantar direction or to adduct when the heel began to lift off of the ground. This visualization was considered to be consistent with abnormal pronation of the midtarsal joint late in the midstance phase of gait and is one of the theoretical compensations for functional hallux limitus.

\section{Results}

A total of 43 subjects (86 feet) were recruited (mean [ $\pm \mathrm{SD}]$ age, $24.0 \pm 7.7$ years; 25 women and 18 men). Two clinicians agreed that functional hallux limitus was present in 53 of the 86 feet and not present in the remaining 33. Function of the midtarsal joint during late midstance was agreed to be abnormal in 62 feet and normal in 24. Forty-five feet were considered to have both a positive functional hallux limitus test result and abnormal midtarsal joint function. Seventeen feet were considered to have a negative test result for functional hallux limitus and abnormal midtarsal joint function, giving a sensitivity for the functional hallux limitus test of 0.72. Eight feet had a positive functional hallux limitus test result and normal midtarsal joint function. Sixteen feet had a negative functional hallux limitus test result and normal midtarsal joint function, giving a specificity for the functional hallux limitus test of 0.66 .

\section{Discussion}

The results of this study show that when the clinical functional hallux limitus test result is positive, abnor-

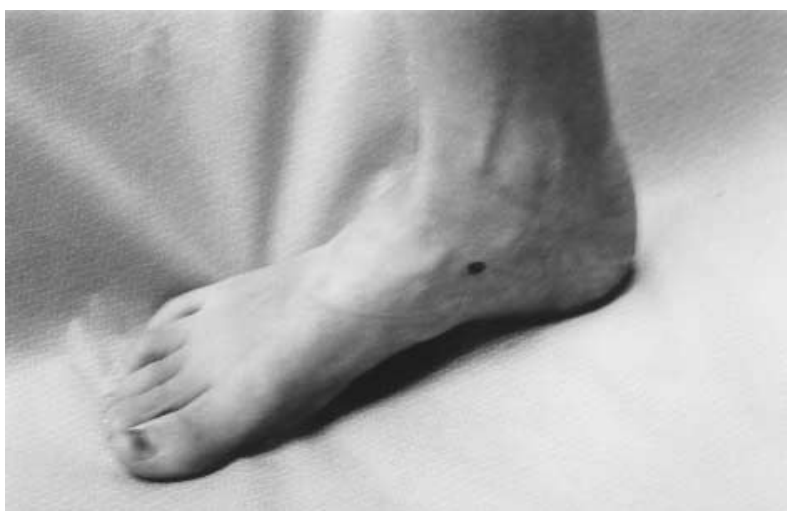

Figure 2. In visual gait analysis, the clinicians observed the movement of a mark placed on the navicular as heel-off begins from the lateral view. 
mal function of the midtarsal joint is present $72 \%$ of the time, and when the test result is negative, there is an absence of abnormal function $66 \%$ of the time. This can be considered good sensitivity and specificity, especially in the context of previously published works ${ }^{8,9}$ showing that other types of static measures are not predictive of dynamic function. These results suggest that this clinical measure may be predictive of dynamic function.

Roukis et $\mathrm{al}^{10}$ demonstrated the importance of first ray plantarflexion in allowing first metatarsophalangeal dorsiflexion. It is this intimate relationship between first ray function and metatarsophalangeal joint motion that forms the basis of the functional hallux limitus test. Kappel-Bargess et al, ${ }^{11}$ while investigating the dynamic function of the windlass mechanism, showed that elevation of the arch and first ray plantarflexion that is related to first metatarsophalangeal joint dorsiflexion and the windlass mechanism occurred in two distinct groups. In one group, there was immediate raising of the arch on first metatarsophalangeal joint dorsiflexion, whereas in the other group, there was a delay in the raising of the arch with first metatarsophalangeal joint dorsiflexion. The group with the delay in the onset of the windlass mechanism, which results in the raising of the arch, also demonstrated greater calcaneal eversion than the group with immediate onset of the windlass mechanism. The theoretical construct of the sagittal plane facilitation model ${ }^{4,6}$ of foot function, based on the concept of functional hallux limitus and its putative compensations, is one explanation for the findings of Kappel-Bargess et al. ${ }^{11}$

Although the results presented here suggest that the clinical functional hallux limitus test can be predictive of abnormal function, the nature of the study design does not imply causality. It cannot be ruled out that the abnormal midtarsal joint pronation observed during gait analysis could be caused by another, unidentified reason and that the clinical functional hallux limitus test result could be positive or negative by coincidence or caused by the same unidentified reason. The more traditional view is that hypermobility of the first ray, secondary to abnormal pronation of the subtalar joint, is the cause of functional hallux limitus. ${ }^{12}$ However, the mechanism by which functional hallux limitus can cause abnormal midtarsal joint pronation is theoretically and biologically possible. Another limitation of this study was the use of visual gait analysis, which has been shown to have poor reliability ${ }^{13}$; however, in this study two clinicians were used, and both agreed on the dichotomous variable of normal versus abnormal function.

\section{Conclusion}

Functional hallux limitus is an underrecognized entity because of the lack of symptoms that occur at the joint. Several possible compensatory mechanisms can occur if functional hallux limitus is present, as indicated by the nonweightbearing clinical functional hallux limitus test result. One compensation is excessive midtarsal joint pronation during the late stance phase as the heel begins to lift, which is observable only from the lateral view during gait. This study has shown good sensitivity (0.72) and specificity (0.66) for the functional hallux limitus test to predict this compensation. Clinicians should consider the possibility of functional hallux limitus when a late midstance "collapse" of the midtarsal joint is noted during gait and should include the clinical test for functional hallux limitus in the routine assessment of a patient's biomechanical function.

\section{References}

1. Dananberg HJ: Functional hallux limitus and its relationship to gait efficiency. JAPMA 76: 648, 1986.

2. DANANBerg HJ: Gait style as an etiology to chronic postural pain: part 1. Functional hallux limitus. JAPMA 83: 433, 1993.

3. DAnAnberg HJ: Gait style as an etiology to chronic postural pain: part 2. Postural compensatory process. JAPMA 83: 615, 1993.

4. Payne CB, Dananberg HJ: Sagittal plane facilitation of the foot. Australas J Podiatr Med 31: 7, 1997.

5. Chapman C: Functional hallux limitus: the essentials. Br J Podiatry 2: 40, 1999.

6. Dananberg HJ: "Sagittal Plane Biomechanics," in Sports Medicine and the Lower Extremity, ed by SI Subotnick, p 137, Churchill Livingstone, New York, 1999.

7. Dananberg HJ: "Lower Back Pain as a Gait Related Repetitive Motion Injury," in Movement: The Pelvis and Low Back Pain-An Interdisciplinary Approach, ed by A Vlemming, V Mooney, T Dorman, p 253, Churchill Livingstone, Edinburgh, 1997.

8. Hamill J, Bates BT, Knutzen KM, et al: Relationship between selected static and dynamic lower extremity measures. Clin Biomech 4: 217, 1989.

9. Knutzen KM, Price A: Lower extremity static and dynamic relationships with rearfoot motion in gait. JAPMA 84: $171,1994$.

10. Roukis TS, Scherer PR, Anderson CF: Position of the first ray and motion of the first metatarsophalangeal joint. JAPMA 86: 538, 1996.

11. Kappel-Bargess A, Woolf RD, Cornwall MW, et Al: The windlass mechanism during normal walking and passive first metatarsophalangeal joint extension. Clin Biomech 13: 190,1998

12. Root ML, ORIEn WP, Weed JH: Normal and Abnormal Function of the Foot, Clinical Biomechanics Corp, Los Angeles, 1977.

13. KeEnan AM, Bach TM: Video assessment of rearfoot movement during walking: a reliability study. Arch Phys Med Rehabil 77: 651, 1996. 